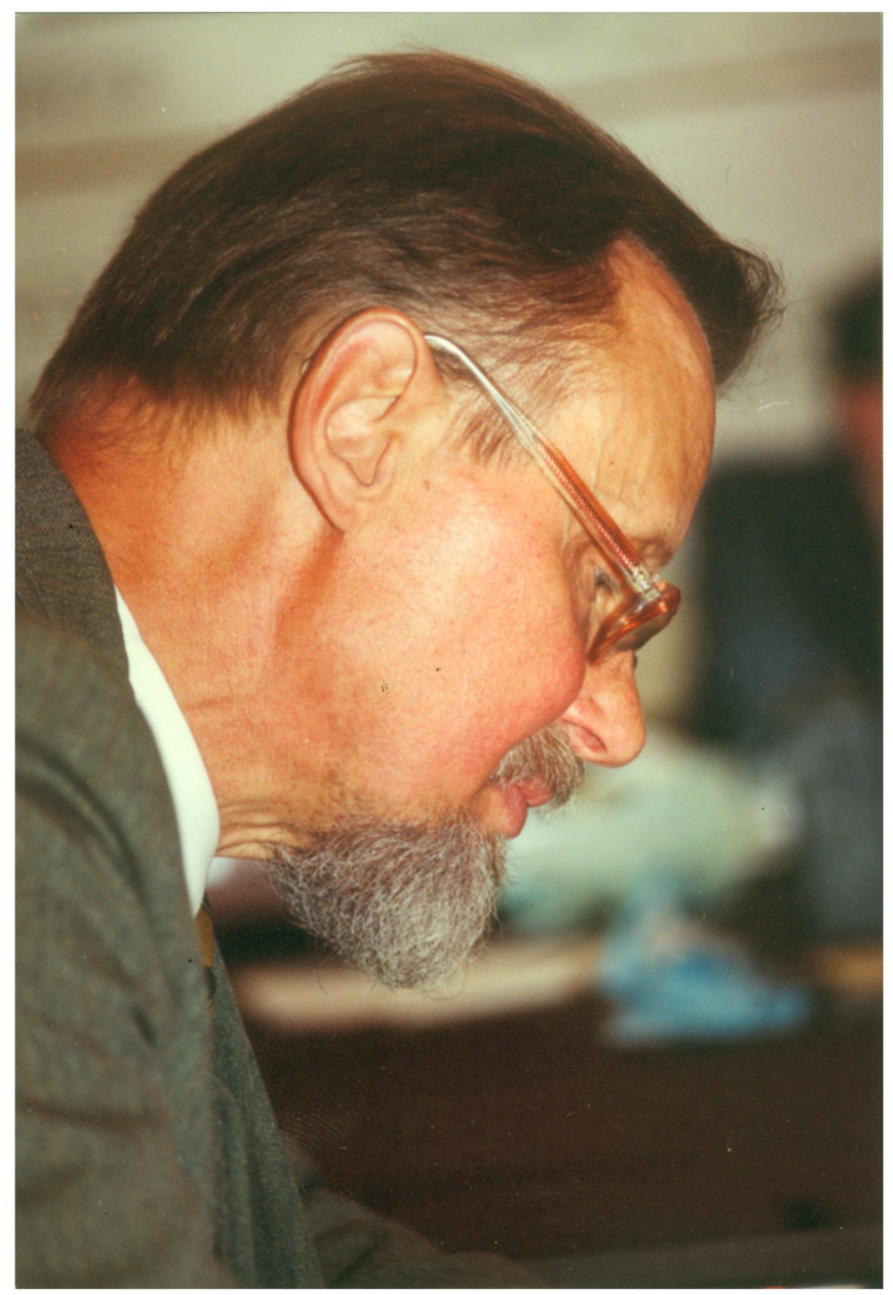

The Editors and the Contributors dedicate this special issue of Fragmenta Faunistica to Professor Przemyslaw Trojan to celebrate his $80^{\text {th }}$ anniversary

Jolanta Wytwer Editor in Chief Fragmenta Faunistica 


\title{
Professor Przemysław Trojan's 80th anniversary
}

\author{
IMPORTANT BIOGRAPHICAL FACTS
}

22 August 1929 Przemysław Trojan is born in Pruszków near Warsaw; 1942-1944 attends underground education classes at W. Górski secondary school in Warsaw; 1943-1944 becomes a Grey Ranks scout, heads squads belonging to the Zawisza Czarny troops (liaison, distribution of underground press, military intelligence);

Aug-Oct 1944 Warsaw Uprising. Co-organizes the first Scout Field Post unit operating in the northern part of the centre of Warsaw, then becomes a liaison officer for the commander of autonomous platoon of the "Kolegium B" unit of the Kedyw military organisation. After the defeat of the Uprising, flees from a train carrying prisoners to a concentration camp; joins underground education again;

1945-1948 attends the Secondary School of Humanities in Gliwice;

1948-1952 studies at the Faculty of Mathematics and Natural Sciences of the University of Warsaw;

1950-1954 research assistant at the Division of Zoology, Warsaw Agricultural University;

1954-1958 organises and heads Dipterology Laboratory, and the PAS Institute of Zoology in Warsaw, attends $\mathrm{PhD}$ studies at the Faculty of Biology, University of Warsaw. Conducts studies of Polish dipterans;

1958 receives a $\mathrm{PhD}$ in Biological Sciences from the Faculty of Biology, Warsaw University for the dissertation "Ecological Niches of Horse Flies in Kampinos Forest";

1958-1963 Assistant Professor at the PAS Institute of Ecology in Warsaw, head of the Institute's branch in Dziekanów Leśny. Studies ecology of animal populations;

1963 receives the degree of doctor habilitatus from the Faculty of Biology, University of Warsaw, for the dissertation "The Concept of Species in the Genus Tabanus L. in the light of Taxonomic Practice";

1963-1968 organises and heads the PAS Division of Agroecology in Turwia (now the PAS Division of Agricultural and Forest Biology), conducts research on protection of the environment and arable land, sustainable production and natural pest reduction in agrocoenoses;

1969-1971 organises the Faculty of Biology at the Silesian University in Katowice and launches degree programmes in ecology and environmental protection there;

1971 becomes Professor Extraordinary;

1972-1981 Deputy Director for Research at the PAS Institute of Zoology in Warsaw. Organises research on the regulation of pest numbers in agrocoenoses, Organises a programme of urban ecology-oriented research on animal populations. Becomes a member of the State Council for Protection of Nature, State Council for Environmental Protection and Ecological Council affiliated with the Ministry of National Defence;

1973-1976 professor at the College of Agriculture and Pedagogy in Siedlce;

1976-1982 Deputy Secretary at the Division of Biological Sciences at the Polish Academy of

Sciences. Organises a co-ordination programme for research in zoology;

1982 becomes Ordinary (Full) Professor; 
1982-1988 Director of the PAS Institute of Ecology in Dziekanów Leśny; co-ordinator of ecological research in Poland. Head of the Committee of Ecology at the Polish Academy of Sciences;

1989-1990 scholarships to Museum für Naturkunde of A. Humboldt University in Berlin and

Muséum National d'Histoire Naturelle in Paris (studies the taxonomy and evolution of blood-sucking Diptera);

1991-1999 Professor at the PAS Institute of Zoology in Warsaw, head of the Laboratory of

Faunal Studies. Carries out research on the diversity, structure and ecological succession of

fauna in typical Polish habitats. Publishes papers in the taxonomy and evolution of Tabanidae;

1991-1999 lecturer at the PhD Division of the Forest Science Faculty of Warsaw Agricultural

University;

1992-2007 President of the "Mazovian Landscapes" Association for Environmental Protection; 1999 Becomes a member of the New York Academy of Sciences;

Since 1999 Treasurer, then head of the "Forester" Grouping of the Union of Warsaw Uprising Insurgents;

Since 2000 Professor emeritus at the PAS Museum and Institute of Zoology in Warsaw;

\section{MAIN RESEARCH INTERESTS}

Taxonomy and zoogeography of Diptera. Professor Trojan's studies of the Polish Diptera have included systematic revisions and monographs on dipterans recorded in Poland published in leading series of Polish zoological publications, i.e. "Keys for Identification of Polish Insects" and "Polish Fauna" (a total of 17 monographs). His habilitation dissertation was concerned with theory of taxonomy, explaining taxonomic consequences of the development of the concept of species from Linnean times until the present and their impact on the genus Tabanus L. ("Koncepcja gatunku w rodzaju Tabanus L. w świetle praktyki taksonomicznej" [The Concept of Species in the Genus Tabanus L. in the light of Taxonomic Practice]). His later research on the taxonomy and evolution of true horse-flies (Tabaninae) in the world encompassed more than 1500 species distributed all over the world. A numerical system for the valuation of morphological characters used for the identification of supraspecific taxa was employed to propose a new division of the subfamily into five tribes and determine new taxonomic scopes for these tribes. Prof. Trojan compiled monographs of supraspecific taxa for the Lepidoselagini, Diachlorini and Tabanini and determined the distribution of several relict species of Lepidoselagini in the Northern Hemishpere, proving that a Holarctic centre had existed alongside the Gondwanan centre and that the traces of the former had not been obliterated by the ice age. Evidence for the existence of this centre has been furnished by the finding of relict species inhabiting isolated sites in mountain areas and in raised bogs. On the basis of the chronology of continental plate drift, Prof. Trojan estimated the time of formation of the tribes of the subfamily Tabaninae. He determined that transitional forms between the Lepidoselagini and Diachlorini can be found in the Australian realm rather than in the Neotropis, as was formerly believed.

Faunal studies. Professor Trojan's research on the Polish dipteran fauna, commenced in 1949, was carried out in areas representing considerable natural value, especially national parks and nature reserves in the Polish lowlands and in mountain areas (Pieniny, Bieszczady). A believer in the use of quantitative methods along with qualitative research in animal studies, he placed particular emphasis on structural analysis of faunal assemblages, thus introducing a new quality in evaluations of the fauna of different habitats. His findings concerning urban fauna are quoted in the literature, including textbooks. The quantitative approach to faunal studies 
has also produced descriptions of the structure of faunas of typical ecosystems that occur in linden-oak-hornbeam and coniferous habitats in Poland. Among comprehensive faunal studies, particular recognition is due to be given to research on the succession of insect communities in the course of development of pine forests in Bialowieża Primeval Forest. The research was carried out by a team of researchers from the PAS Institute of Zoology and the results were published in a number of monographs.

Ecology. Among important early achievements of Professor Trojan in ecology are studies elucidating the ecological mechanisms underlying the distribution and activity of horse-flies and the intensity of fly attacks of mammals. These results were included by $\mathrm{M}$. Leclercq in his world-wide monograph "Les mouches nuisibles". Population- and bioenergy-oriented studies of rodents resulted in precise determination of the consumption of plant mass by Microtus arvalis Pall., the most important agricultural pest in Poland. A new model for evaluation of the maintenance cost of this pest developed by Prof. Trojan was positively reviewed in the world literature. Studies of the Colorado potato beetle were concerned with the dynamics and biocoenotic reduction of the pest's abundance and provided a new method for estimating consumption based on energy balance associated with the successive developmental stages of this beetle. One important outcome of this approach was the finding that, 15 years after an invasion, the natural reduction of the beetle is $95-98 \%$, and its consumption of potato leaves by is often below the harm threshold. A new model for the evaluation of energy balance in animals was also developed based on the assessment of the effect of cumulative factors influencing metabolism rates. His studies of the entomocoenoses of arable fields were helpful in elucidating the effect of clumps of trees growing in fields on the spatial distribution of pests, predators and parasites. These clumps function as winter refuges for the field fauna and centres of aggregation of useful animal species. Studies in agroecology and ecology of urban fauna lay the foundation for a unique concept of ecosystem homoeostasis. The concept demonstrated that the maintenance of ecological balance in an ecosystem depends on the interaction of four subsystems, namely, those of producers, biotrophs, saprotrophs and macrotrophs. Of these four, the biotrophic subsystem is the most developed one and includes numerous, often structurally complex, food chains. This work was also published in English.

Apart from his research activities, Prof. Trojan was also involved in teaching, being the advisor of $3 \mathrm{MSc}$ projects, 12 doctorates, and the proponent of 3 habilitation dissertations. He also gave lectures in ecology (Silesian University, $\mathrm{PhD}$ Division of Warsaw Agricultural University, PAS College) and in environmental protection and evolutionism (College of Agriculture and Pedagogy in Siedlce).

Professor Trojan is a member of the Polish Society of Entomology, and sits on Editorial Boards of the Polish Journal of Ecology, Fragmenta Faunistica, and the Polish Journal of Entomology. The Polish Society of Entomology awarded him the Golden Badge, PSE Medal of Merit, and an Honorary Membership. Official honours for his work have included awards from the PAS Scientific Secretary (5 times), Minister of Agriculture, Minister of Higher Education and Science, and Minister of Environmental Protection. He has also received the Officer's Cross of the Order of Restitution of Poland, the Warsaw Uprising Cross, Home Army Cross, and the Polish Scouting Association Cross of Merit with swords.

\section{Dr Waldemar Mikolajczyk ${ }^{1}$}

\footnotetext{
${ }^{1}$ Based on archives of the PAS Museum and Institute of Zoology and information provided by Prof. Trojan himself
} 


\section{BIBLIOGRAPHY OF PRZEMYSŁAW TROJAN}

\section{3}

Trojan P. 1953. O czym mówią badania geologiczne i paleontologiczne. Wszechnica Radiowa, Kurs I, Cykl Przyroda, Skrypt 6, Wykład 28: 174-182, 1952/1953.

TRojaN P. 1953. Dowody jedności świata organicznego. Wszechnica Radiowa, Kurs I, Cykl Przyroda, Skrypt 6, Wykład 29: 182-189, 1952/1953

TROJAN P. 1953. Ewolucja zwierząt kręgowych. Wszechnica Radiowa, Kurs I, Cyk1 Przyroda, Skrypt 7, Wykład 30: 193-198. 1952/53.

TROJAN P. 1953. Teoria ewolucyjna Darwina. Wszechnica Radiowa, Kurs II, Cyk1 Materializm Dialektyczny i Historyczny, Skrypt 5, Wykład 8: 57-63. 1952/53.

TroJan P. 1953. Twórczy darwinizm radziecki. Wszechnica Radiowa, Kurs II, Cykl Materializm Dialektyczny i Historyczny, Skrypt 5, Wyklad 9: 65-72, 1952/1953.

TROJAN P. 1953. Z zagadnień doboru naturalnego. Wiedza i Życie, 8: 535-538.

BEAŻEJEWICZ W., JANION M., KOWALSKA, K. \& TROJAN P. 1953. Bibliografia prac z dziedziny ewolucjonizmu. Warszawa, 1: 1-35, 2: 1-54.

\section{4}

LASMAN M., TROJAN P. \& TRoJANowa R. 1954. Kilka uwag na temat artykułu S. Ehrlicha i W. Kaczmarka. Kosmos A, 3, 1: 71-73.

TROJAN P. 1954. Wlaściwości żywej materii. In: "Wszechświat, Życie, Człowiek”, Książka i Wiedza, Warszawa: 172 180, 2nd ed., 1955.

TROJAN P. 1954. Odżywianie się zwierząt. In: "Wszechświat, Życie, Człowiek", Książka i Wiedza, Warszawa: 193 201, 2nd ed., 1955.

Trojan P. 1954. Oddychanie. In: "Wszechświat, Życie, Człowiek", Książka i Wiedza, Warszawa: 202-210, 2nd ed., 1955.

TROJAN P. 1954. Wydalanie w świecie zwierząt. In: "Wszechświat, Życie, Człowiek", Książka i Wiedza, Warszawa: 211-219, 2nd ed., 1955.

TROJAN P. 1954. Organizm i środowisko. In: "Wszechświat, Życie, Człowiek", Książka i Wiedza, Warszawa: 220 226, 2nd ed. 1955.

TROJAN P. 1954. O czym mówią badania geologiczne i paleontologiczne. In: "Wszechświat, Życie, Człowiek", Książka i Wiedza, Warszawa: 229-240, 2nd ed., 1955.

TROJAN P. 1954. Dowody jedności świata organicznego. In: "Wszechświat, Życie, Czlowiek", Książka i Wiedza, Warszawa: 241-248, 2nd. ed., 1955.

TROJAN P. 1954. Ewolucja zwierząt kręgowych. In: "Wszechświat, Życie, Człowiek", Książka i Wiedza, Warszawa: 249-260, 2nd. ed., 1955.

TROJAN P. 1954. Zmienność - podstawowa cecha żywej materii. In: "Wszechświat, Życie, Człowiek", Książka i Wiedza, Warszawa: 261-269, 2nd ed., 1955.

\section{5}

TROJAN P. 1955. Właściwości żywej materii. In: "Wszechświat, Życie, Człowiek", Książka i Wiedza, Warszawa, 3rd revised ed.: 219-227.

TROJAN P. 1955. Odżywianie się zwierząt. In: "Wszechświat, Życie, Czlowiek", Książka i Wiedza, Warszawa, 3rd revised ed.: $241-251$.

TROJAN P. 1955. Oddychanie istot żywych. In: "Wszechświat, Życie, Człowiek", Książka i Wiedza, Warszawa, wyd. 3. zmienione: $252-260$.

TROJAN P. 1955.. Wydalanie w świecie zwierząt. In: "Wszechświat, Życie, Człowiek", Książka i Wiedza, Warszawa, 3rd revised ed.: $261-269$.

TROJAN P. 1955. Organizm i środowisko. In: "Wszechświat, Życie, Czlowiek", Książka i Wiedza, Warszawa, 3rd revised ed: $270-276$.

TroJAN P. 1955. Dowody jedności świata organicznego. In: "Wszechświat, Życie, Czlowiek", Książka i Wiedza, Warszawa, 3rd revised ed.: 301-307.

TROJAN P. 1955. Zmienność - podstawowa cecha żywej materii. In: "Wszechświat, Życie, Człowiek", Książka i Wiedza, Warszawa, 3rd revised ed.: $308-318$.

TROJAN P. 1955. Ewolucja roślin. In: "Wszechświat, Życie, Człowiek", Książka i Wiedza, Warszawa, 3rd revised ed.: 319-332

TROJAN P. 1955. Ewolucja zwierząt bezkręgowych. In: "Wszechświat, Życie, Człowiek", Książka i Wiedza, Warszawa, 3rd revised ed.: 333-344.

TROJAN P. 1955. Ewolucja zwierząt kręgowych. In: "Wszechświat, Życie, Człowiek", Książka i Wiedza, Warszawa, 3rd revised ed: $345-358$.

Trojan P. 1955. Tabanidae okolic Warszawy (Diptera). Fragmenta Faunistica, 7, 4: 180-207. 
1956

TrojAN P. 1956. Cyrtidae. Klucze do oznaczania owadów Polski, PWN, Warszawa, 28, 23: 17 pp.

TROJAN P. 1956. Erinnidae. Klucze do oznaczania owadów Polski, PWN, Warszawa, 28, 19: 21 pp.

TROJAN P. 1956. Omphralidae. Klucze do oznaczania owadów Polski, PWN, Warszawa, 28, 26: 15 pp.

TROJAN P. 1956. Oncodes reginae sp.n. oraz uwagi o gatunkach europejskich z rodziny Cyrtidae (Diptera). Annales Zoologici, 16, 8: 73-79.

TrojAN P. 1956. Uwagi o taksonomii kilku europejskich gatunków z rodzaju Omphrale Meig. (Diptera, Omphralidae). Annales .Zoologici, 16, 11: 147-156.

Trojan P. 1956. Metodyka badań ekologicznych Tabanidae (Diptera). Ekologia Polska B, 2, 1: 41-46.

TROJAN P. 1956. Zagadnienie środowiska gatunku jako problem metodologiczny. Ekologia Polska B, 2, 4: 323 -330.

1957

TROJAN P. 195 Muchówki (Diptera) - część ogólna. Klucze do oznaczania owadów Polski, PWN, Warszawa, 28, 1: $145 \mathrm{pp}$.

1958

TROJAN P. 1958. The ecological niches of certain species of horse-flies (Diptera, Tabanidae) in the Kampinos forest near Warsaw. Ekologia Polska A, 6, 2: 53-129.

TROJAN P. 1958. Muchy i człowiek. PWN, Warszawa, 125 pp.

1959

TrojaN P. 1959. Ślepaki - Tabanidae. Klucze do oznaczania owadów Polski, PWN, Warszawa, 28, $21: 69$ pp.

Trojan P. 1959. Distributional notes on Ethiopian species related to Tabanus L. (Diptera, Tabanidae). Fragmenta Faunistica, 8, 13: 223-226.

\section{0}

WOJCIECHOWSKA B. \& TROJAN P. 1960. An attempt at analysing the habitat of Viviparus fasciatus (Müll). Ekologia Polska A, 8, 12: 281-302.

Trojan P. 1960. Ślepaki (Diptera, Tabanidae) Niecki Nidziańskiej. Fragmenta Faunistica, 8, 18: 285-291.

\section{1}

TROJAN P. 1961. Lowiki (Diptera, Asilidae) stanowisk kserotermicznych Polski. Fragmenta Faunistica, 9, 10: 109-121.

\section{2}

TROJAN P. 1962. Analysis of the species concept in the genus Tabamus L. (Diptera) as shown by taxonomic practice.

Ekologia Polska A, 10, 6: 123-229.

TrojaN P. 1962. Odiniidae. Klucze do oznaczania owadów Polski, PWN, Warszawa, 28, 54: 1-9.

Trojan P. 1962. Clusiidae. Klucze do oznaczania owadów Polski, PWN, Warszawa, 28, 55: 10-30.

TROJAN P. 1962. Anthomyzidae. Klucze do oznaczania owadów Polski, PWN, Warszawa, 28, 56: 31-42.

TrojaN P. 1962. Opomyzidae. Klucze do oznaczania owadów Polski, PWN, Warszawa, 28, 57: $43-60$.

TROJAN P. 1962. Tethinidae. Klucze do oznaczania owadów Polski, PWN, Warszawa, 28, 58: 61-68.

\section{3}

TROJAN P. 1963. Stratiomyidae. Klucze do oznaczania owadów Polski, PWN, Warszawa, 28, 22: 72 pp.

OPUSZYŃSKI K. \& TROJAN P. 1963. Distribution of burrows and elements of the population structure of small forest rodents. Ekologia Polska A, 11, 14: 339-352.

TROJAN P. \& WOJCECHOWSKA B. 1963 New method of estimation of rodent residence in investigations performed by the CMR technique. Bulletin de l'Académie Polonaise des Sciences, Cl. II., 9, 8: 375-378.

PETRUSEWICZ K.\& TROJAN P. 1963. The influence of the size of the cage on the numbers and density of a self-ranging population of white mice. Ekologia Polska A, 11, 28: 611-614.

\section{4}

TROJAN P. \& WojCIECHOWSKA B. 1964. Studies on the residency of small forest rodents. Ekologia Polska A, 12, 3: 33-50.

TROJAN P. \& WoJCIECHOWSKA B. 1964. The distribution of captures of small rodents and its causes. Ekologia Polska A, 12, 21: 369-378.

Trojan P. 1964. Muchówki pożyteczne. Zeszyty Problemowe Postępów Nauk Rolniczych, 45: 117-120.

PETRUSEWICZ K. \& TROJAN P. 1964. Źródla wspólezesnej koncepcji gatunku. Kosmos, A, 13, 4: 283-295.

\section{5}

TROJAN P. 1965. Intrapopulation relations and regulation of numbers in small forest rodents. Ekologia Polska A, 13, 11: $143-168$. 


\section{6}

TrojaN P. \& WojCIECHOWSKa B. 1966. The specific distinction of Chrysozona pluvialis (L.) and Ch. hispanica (Szil.) (Diptera, Tabanidae) in Poland. Annales Zoologici, 23, 23: 525-534.

TROJAN P. 1966. Organizacja i problematyka Stacji Agroekologicznej PAN w Turwi w latach 1963-1966. Kosmos A, $15,5: 552-556$.

1967

Trojan P. 1967. Bujanki - Bombyliidae (Diptera). Klucze do oznaczania owadów Polski, PWN, Warszawa, 28, $24: 44$ pp.

TROJAN P. 1967. Investigations on production of cultivated fields In: PETRUSEWICZ K. (ed.), Secondary Productivity of Terrestrial Ecosystems, Warszawa-Kraków: 545-561.

CHLODNY J, GROMADZKA J. \& TROJAN P. 1967. Energetic budget of development of the Colorado beetle Leptinotarsa decemlineata Say (Coleoptera, Chrysomelidae). Bulletin de 1'Académie Polonaise des Sciences, Cl. II., 15, 12: $743-747$.

GROMADZKA J. \& TROJAN P. 1967. Comparison of the usefulness of an entomolical net, photo-eclector and biocenometer for investigation of entomocenoses. Ekologia Polska, 15, 24: 505-529.

HeRBICH M. \& TROJAN P. 1967. Stability in the caloric equivalent of the potato. Bulletin de l'Académie Polonaise des Sciences, C1. II., 15, 12: 739-741.

KARG J. \& TROJAN P. 1967. Redukeja naturalna i szkodliwośé stonki Leptinotarsa decemlineata Say w Polsce. Konference o skudcich okopanin III, Praha, 7-23.

TROJAN P. \& WOJCIECHOWSKA B. 1967. The reaction of small rodents to a new object and estimate of population numbers. Ekologia Polska, A, 15, 36: 727-736.

TROJAN P. \& WOJCIECHOWSKA B. 1967. Resting metablism rate in the european common vole - Microtus arvalis (Pall.) in different ambient temperatures. Ekologia Polska, A, 15, 43: 803-810.

TROJAN P. \& WOJCIECHOWSKA B. 1967. Resting metabolism rate during pregnancy and lactation in the european common vole - Microtus arvalis (Pall.). Ekologia Polska, A, 15, 44: 811-817.

\section{8}

TRojaN P. 1968. Agrocenoza jako biologiczny układ produkcyjny. Polskie Pismo Entomologiczne, 38, 3: $647-655$.

TROJAN P. 1968. Egg reduction of the Colorado beetle (Leptinotarsa decemlineata Say) as a hunger-dependent reaction. Ekologia Polska, A, 16, 6: 171-183.

TROJAN P. 1968. Estimated food consumption by the Colorado beetle (Leptinotarsa decemlineata Say) under conditions of natural reduction. Ekologia Polska, A, 16, 18: 385-393.

KARG J. \& TROJAN P. 1968. Fluctuations in numbers and reduction of the Colorado beetle (Leptinotarsa decemlineata Say) in natural conditions. Ekologia Polska, A, 16, 5: 147-169.

TROJAN P. \& WOJCIECHOWSKA B. 1968. The effect of huddling on the resting metabolism rate in european common vole (Microtus arvalis Pall.). Bulletin de 1'Académie Polonaise des Sciences, Cl. II., 16, 2: 107-109.

TROJAN P. \& WOJCIECHOWSKA B. 1968. The influence of darkness on the oxygen consumption by nesting individuals of the European Common Vole - Microtus arvalis (Pall.). Bulletin de 1'Académie Polonaise des Sciences, Cl.II., 16, 2: 111-112.

\section{9}

TROJAN P. 1969. Gleba jako biologiczny układ biologiczny. Międzynarodowy Uniwersytet Radiowy.

TROJAN P. 1969. An ecological model of the costs of maintenance of Microtus arvalis (Pall.). In: PETRUSEWICZ K. \& RYSZKOWSKI L.(eds), Energy flow through small mammal populations, pp. 113-122. PWN, Warszawa, 298 pp.

TROJAN P. 1969. Energy flow trough a population of Microtus arvalis (Pall.) in an agrocenosis during a period of mass occurrence. In: PETRUSEWICZ K. \& RYSZKOWSKi L.(eds), Energy flow through small mammal populations, pp. 267-279. PWN, Warszawa, $298 \mathrm{pp.}$

TROJAN P. \& WOJCIECHOWSKA B. 1969. Ecological model and tables of the daily costs of maintenance /DEB/ of Microtus arvalis (Pall.). Ekologia Polska, A, 17,17: 313-342.

\section{0}

TroJAN P. 1970. Lowiki - Asilidae. Klucze do oznaczania owadów Polski, PWN, Warszawa, XXVIII, 27,89 pp.

TrojaN P. 1970. Thereva thermopila sp. nov. (Diptera, Therevidae) from Poland. Bulletin de l'Académie Polonaise des Sciences, Cl.II., 18, 5: 283-286.

TROJAN P. 1970. Therevidae. Klucze do oznaczania owadów Polski, PWN, Warszawa, XXVIII, 25, 28 pp.

GROMADZKI M. \& TROJAN P. 1971. Estimation of population density in Microtus arvalis (Pall.) by three different methods. Annales Zoologici Fennici, 8: 54-59. 
1972

Trojan P. 1972. Ekologia. Częśś I. Wstęp do ekologii. Bioklimatologia ekologiczna. Katowice. Wyd. Uniwersytetu Śląskiego, $149 \mathrm{pp}$.

TROJAN P. 1972. Zadania i możliwości ekologii w zakresie problematyki "Człowiek i środowisko". Wiadomości Ekologiczne, 18, 3: 282-293.

TROJAN P. 1972. Zagadnienie kierowania ekosystemami. Kosmos A, 4, 117: 409-416

1974

TrojaN P. 1974. Głos w dyskusji nad zagadnieniem ochrony środowiska. Zeszyty Problemowe Postępów Nauk Rolniczych, 155: 149-152.

Trojan P. 1974. Przegląd faunistyczny Stratiomyidae (Diptera) Polski. Fragmenta Faunistica, 20, 2: 15-27.

TROJAN P. 1974. Zagadnienie homeostazy ekosystemów. Zeszyty Problemowe Postępów Nauk Rolniczych, 155: 39-51.

ANDRZEJEWSKI R. \& TROJAN P. 1974. Osiagnięcia i perspektywy ekologii populacji. Wiadomości Ekologiczne, 20, 2 95-106.

1975

TROJAN P. 1975. Ekologia ogólna. PWN, Warszawa, 419 pp.

TROJAN P. 1975. Cele i zalożenia badań zoocenologicznych osiedla Białolęka Dworska w Warszawie. Fragmenta Faunistica, 26, 1: 10-15.

Trojan P. 1975. Pierwszy Międzynarodowy Kongres Ekologiczny. Kosmos, 24,4: 406-412.

BańKOWSKa R., KIERYCH E., MIKOlAJCZYK W., PALMOWSKA J. \& TROJAN P. 1975. Aphid-aphidophage community in Alfalfa cultures (Medicago sativa L.) in Poland. 1. Structure and phenology of the community. Annales Zoologici, 32, 14: 299-345.

PISARSKI B. \& TROJAN P. 1975. Zoocenozy obszarów zurbanizowanych. Wiadomości Ekologiczne 22, 4 : 338-344.

1976

TROJAN P. 1976. Ekosystemy wobec presji czlowieka. Czlowiek i Światopogląd, 7/8, 132-133: 35-45.

TROJAN P. 1976. Homeostaza agroekosystemów a perspektywy walki biologicznej ze szkodnikami. Wiadomości Ekologiczne, 22, 3: 238-242.

Trojar P. 1976. Parancala kibic gen. nov. sp. nov. a New Horse-fly of African Origin in Oriental Fauna (Diptera, Tabanidae). Bulletin de l'Académie Polonaise des Sciences, Cl. II., 24, 3: 133-137.

TROJAN P. 1976. Przedmowa do wydania polskiego. In: STUGREN B.: Zasady ekologii ogólnej, Warszawa, PWN: 7-8.

TrojaN P. 1976. R. Rozkošny. The Stratiomyidae (Diptera) of Fennoscandia and Denmark. Fauna Entomologica Scandinavica. Vol. 1, Scandinavian Science Press Ltd., Gadstrup (Denmark), 1973, 140 pp., 456 ff., 1 t.. Polskie Pismo Entomologiczne, 46, 1: 201-203.

SzELEGIEWICZ H. \& TROJAN P. 1976. Okhrana nasekomykh v Polshe. Proc. III Conf. Prot. Insects, Erevan: 150-156.

PISARSKI B. \& TROJAN P. 1976 Rola zoocenoz w środowisku zurbanizowanym. In: CZARNOTA K. \& ŻUKOWSKAWIESZCZEK D. (eds), Ekologiczne Problemy Miasta, pp. 55-62. Wydawnictwo SGGW, Warszawa.

PISARSKI B. \& TROJAN P. 1976 Wpływ urbanizacji na entomofaunę. In: SANDNER H. (ed.), Entomologia a ochrona środowiska, pp. 65-75. PWN, Warszawa:, $252 \mathrm{pp}$

\section{7}

TrojaN P. 1977. Przyroda wzorem. Życie i Nowoczesność, 376: 1-4.

Trojan P. 1977 Tabanus castellamus Strobl, 1906 (Diptera, Tabanidae) a species distinct in the Europaean Fauna. Bulletin de l'Académie Polonaise des Sciences, Cl. II., 24, 12(1976): 747-750.

TROJAN P. 1977. Znaczenie systematyki w badaniach agrocenoz. Biuletyn Informacyjny Polskiego Towarzystwa Entomologicznego, 19: 17-21.

\section{8}

Bańkowska R., MikolajczyK W., PALMOWSKa J. \& TROJAN P. Aphid-aphidophage community in Alfalfa cultures (Medicago sativa L.) in Poland. Part 3. Abundance regulation of Acyrthosiphon pisum (Harr.) in a chain of oligophagous predators. Annales Zoologici, 34, 3: 39-77.

Trojan P. 1978. Pojęcie równowagi ekologicznej ekosystemów. Kosmos, A, 27, 2: 139-149.

TROJAN P. 1978. Pierwsze spotkanie ewolucjonistów i teoretyków biologii krajów socjalistycznych. Kosmos, A, 27, 2 221-223.

TroJAN P. 1978. Równowaga ekologiczna w przyrodzie. Biologia w Szkole, 31, 2: 58-60.

TROJAN P. 1978. J. A. Chmurzyński - W poszukiwaniu istoty życia. Kosmos A, 27, 4: 437-440.

TROJAN P. 1978. Redukcjonista na poletku ekologii. Wiadomości Ekologiczne, 24, 3: 272-276.

TROJAN P. 1978. Problemy żywienia a problemy ochrony środowiska czlowieka we wspólezesnej cywilizacji. In: „Ekologia Żywienia Człowieka", pp. 5-15. Ossolineum, Warszawa. 
TROJAN P. 1979. Filozofia przyrody warszawskiej szkoly ekologicznej. Kosmos, A, 28, 1: 57-66.

TROJAN P. \& ZOLICH E. 1979. Sympozjum "Homeostaza ekosystemów lądowych", Jabłonna, 6-11.06.1978 r. Kosmos,

A, 28, 1: 119-120.

Trojar P. 1979. Tabanidae, ślepaki (Insecta: Diptera). Fauna Polski, Warszawa, 309 pp.

TROJAN P. 1979. Units of the homeostatic organisation of terrestial ecosystems. Memorabilia Zoologica, 32: 13-23.

TrojaN P. 1979. Drugi Międzynarodowy Kongres Ekologiczny. Kosmos, A, 28: 234-238.

TROJAN P. 1979. Badania nad ogólną teorią biocenologii w ramach problematyki ochrony ekosystemów i krajobrazu realizowanej przez kraje RWPG. Kosmos, A, 28, 4: 379-386.

TROJAN P. 1979. Program badawezy krajów RWPG "Wspólnymi silami do jednego celu". Trybuna Ludu, 207.

TROJAN P. 1979. Stan i potrzeby badań ekologicznych w Polsce w zakresie gospodarki żywnościowej. Nauka Polska, 27, 10: 37-41.

TROJAN P. 1979. Co nas gryzie? Kulisy: 3.

\section{0}

TROJAN P. 1980. Współczesne problemy faunistyki. .Wiadomości Entomologiczne, 1, 1: 3-14.

TROJAN P. 1980. Association structure as indicator of the ecosystem homeostasis. Kongress- und Tagungsberichte der Martin-Luther Universitaet, Halle-Wittenberg, 5: 50-56.

Trojan P. 1980. Homeostaza ekosystemów. Ossolineum, Wroclaw, 149 pp.

Trotan P. \& URBanEK A. 1980. Nauki biologiczne w Polsce w latach 1971-1979. In: "Nauka a rozwój kraju", Ossolineum, Warszawa : 69-84.

StENSETH N.CHR., FRAMSTAD E., MigUla P., TROJAN P. \&WOJCECHOWSKa-TROJAN B. 1980. Energy models for the european common vole (Microtus arvalis Pall.): energy as limiting resource for reproductive output. Oikos, 34: 1-22.

TROJAN P. 1980. Nie gwałcąc natury. Kontakty, 5, 10:6.

\section{1}

TROJAN P. 1981. Cele i założenia badań zoocenologicznych osiedla Bialołęka Dworska w Warszawie. Fragmenta Faunistica, 26, 1:9-15.

TROJAN P. 1981. Urban fauna: faunistic, zoogeographical and ecological problems. Memorabilia Zoologica, 34: 3-12.

TROJAN P. 1981. Diptera Tabanomorpha of Warsaw and Masovia. Memorabilia Zoologica 35: 3-31.

Tejchma A., Zieleński J., Grabowska B., Gaęiski B., Kuszka W., Mejro Cz., Ryszkowski L. \& Trojan P. 1981. Wyżywić nie niszcząc, czyli o rolnictwie biologicznym i ekologicznym. Problemy, 7/8: 44-46, 65-68.

\section{2}

TROJAN P. 1982. Las Bielański w Warszawie. In: BAUM T. \& TROJAN P. (eds), Las Bielański w Warszawie. Rezerwat przyrody, p. 7-19. PWN, Warszawa.

BAUM T., LASZEK CZ. \& TROJAN P. 1982. Ochrona i restytucja Lasu Bielańskiego. Las Bielański w Warszawie. In: BAUM T. \& TROJAN P. (eds), Las Bielański w Warszawie, rezerwat przyrody, pp. PWN, 262-281. Warszawa.

TROJAN P. 1982. Formowanie fauny zespołu osiedli mieszkaniowych w Białołęce Dworskiej w Warszawie. Człowiek i Środowisko, 6, 3-4: 435-453.

TROJAN P. 1982 Zoological investigations connected with the Bialolęka Dworska project in Warsaw. In: "Zwierzęta w świecie zurbanizowanym". Ossolineum, Warszawa: 141-154.

TROJAN P. Górska D. \& WEGNER, E. 1982 Processes of synanthropization of competitive animal associations. Memorabilia Zoologica, 37: 125-135.

\section{3}

KACZMAREK W. \& TROJAN P. 1983. Nekotorye voprosy ékologii landshafta v svyazi s optimalizatsei landshaftnykh struktur na primiere Kampinoskogo Natsjonal'nogo Parka. Ékol. Koop., Prilozhenie 2: 82-85

TROJAN P. 1983. Spotkanie Komitetów Narodowych krajów socjalistycznych uczestniczących w programie UNESCO "Człowiek i Biosfera". Nauka Polska, 31, 5: 121-122.

TROJAN P. 1983. IV-te posiedzenie koordynacyjne Komitetów Narodowych krajów socjalistycznych programu UNESCO „Człowiek i Biosfera", 11-15 kwiecień, Klink, NRD. Kosmos A, 32, 3: 475-478.

\section{4}

TROJAN P. 1984. Ecosystem homeostasis. PWN, Warszawa, Dr W. Junk Publishers, 132 pp.

Trojar P. 1984. Polish ecology: A decade after the 2nd Congress of Polish Science. Intecol Newsletter, 14, 5: 1, 4.

TROJAN P. 1984. Present and prognosticated fauna of the housing estate Białolęka Dworska, Warsaw. Memorabilia Zoologica, 40, 166+2 pp.

TROJAN P. 1984. Realizacja postanowień i uchwal II Kongresu Nauki Polskiej w zakresie nauk ekologicznych. Wiadomości Ekologiczne, 30, 3: 235-247.

TROJAN P. 1984. V spotkanie Komitetów Narodowych Krajów Socjalistycznych programu UNESCO "Czlowiek i Biosfera", (Sofia 23-27.IV 1984 r., Nauka Polska, 32, 5-6: 177-179. 
PINOWSKA B., PINOWSKi J. \& TROJAN P. 1984. III Europejskie Sympozjum Ekologiczne "Współzależności między roślinami a zwierzętami" (Lund, 22-26 VIII 1983 r.) Wiadomości Ekologiczne, 30, 2: 199-202.

TROJAN P. 1984. Wkład profesora Kazimierza Petrusewicza (1906-1982) w rozwój teorii gatunku. Wiadomości Ekologiczne, 30, 1: 13-18.

ODECHOWSKA, Z. \& TROJAN P. 1984. Wolę być, uwagi - refleksje - opinie. Na przelaj, 31: 2-3 (29.07. 1984)

TROJAN P. 1984. Środowisko Polski w perspektywie ekologicznej. Materialy Konferencji „Czlowiek-ŚrodowiskoZdrowie", Jabłonna, 7 pp.

\section{5}

TROJAN P. 1985. Bioklimatologia ekologiczna. PWN, Warszawa, 255 pp.

TrojaN P. 1985. Ekologia i pieniądze. Argumenty, 46 (1419): 4.

TROJAN P. 1985. Piąta konferencja krajów socjalistycznych uczestniczących w programie UNESCO "Człowiek $i$ biosfera", Sofia 1984 r. Kosmos, 34, 2: 375-376.

TROJAN P. 1985. Program naukowy Instytutu Ekologii PAN: kierunki badań i wyniki. Kosmos, 34, 2: 355-373.

\section{6}

Trojan P. 1986. The programme of scientific investigations in Polish Biosphere Reserves. Proc. European MaB Conference on Biosphere Reserves and Ecological Monitoring, České Budějovice: 107-110.

TROJAN P. 1986 Razrabotki po ekologiczna biotechnologija v Instituta po ekologija pri Polskata Akademija na Naukite. In: "Ekologizacja'85", Sofia: 29-35.

Trotan P. 1986. 35-lecie Państwowego Wydawnictwa Naukowego. Wiadomości Ekologiczne, 32, 3: 315-316.

TROJAN P. 1986 Reforma gospodarcza a ochrona środowiska. Materialy sesji "Prawna Ochrona Środowiska", Warszawa: 124-152.

TROJAN P. 1986. Ekologicka polityka socialistickych zemi. In: "Socialismus a globálni problemy soucastnosti, II: 183-196".

TROJAN P. 1986. Nic za darmo. Kulisy, 57 (11984): 3-9.

TROJAN P. 1986. VI Konferencja Komitetów Narodowych "Czlowiek i Biosfera" Krajów Socjalistycznych 17-22 marca 1986, Brno. Kosmos, 35, 3(192): 465-467.

\section{7}

TROJAN P. 1987. Ekologia i rozwój. Zarządzanie, 14, 5: 8-9.

TROJAN P. 1987. Ekologia jako nauka interdyscyplinarna. Biologia w Szkole, 40, 3: 131-138.

TROJAN P. 1987. Ekologiczny problem globalny. Materiały Studialne Komitetu Badań nad Krajami Światowego Systemu Socjalistycznego, Ossolineum, Wrocław, 1: 3-23.

TrojaN P. 1987. Redescription de Chalybosoma cyaneoviridis (Macquart, 1850) de Tasmanie. Revue Française d'Entomologie, 9, 4: 176-178.

TROJAN P. 1987. Ékologiya kak interdisciplinarnaya nauka. Bjul. Socjalno-ékonomicheskie, organizatsionno pravovye i pedagogicheskie aspekty okhrany okruzhayushcheï sredy. Izd. Instituta Okruzhayushcheï Sredy, Warszawa, 17: 9-19.

\section{8}

NEY R. \& TROJAN P. 1988. Przyspieszenie rozwojowe krajów wspólnoty socjalistyeznej a rozwiąywanie problemów globalnych (energetycznego, żywnościowego i ekologicznego). Materiały Studialne Komitetu Badań nad Krajami Światowego Systemu Socjalistycznego przy Prezydium PAN, 2: 129-160.

TROJAN P. 1988. Ékologicheskaya bioklimatologiya. "Wysshaya Shkola", Moskva, 207 pp.

\section{9}

TROJAN P. 1989. 1. Czym jest współczesna biologia. 2. Organizmy a środowisko. In: "Biologia", PWRiL: 10-12, 452-478.

DOBROWOLSKI K. A., JANKOWSKA-KLAPKOWSKA A., PRANDECKA B. \& TROJAN P. 1989. Polityka ekologiczna wybranych krajów socjalistycznych (synteza ekspertyzy). Materiały Studialne Komitetu Badań nad Krajami Światowego Systemu Socjalistycznego przy Prezydium Polskiej Akademii Nauk, 3:103-176.

Trojan P. 1989. Bug (Heteroptera) associations in the agricultural landscape of Great Poland. Ekologia Polska, 37, 1-2: $135-155$.

TROJAN P. 1989. Faktorialnaja ekologia. "Vyssha Shkola", Kiev, 232 pp.

\section{0}

TrojaN P. 1990. Hybomitra (Sipala) ségui, une nouvelle espèce endemique de la Région mediterranéenne (Diptera: Tabanidae). Annales de la Société entomologique de France, (N.S.), 26, 3: 427-430.

TROJAN P. 1990. Environmental policy in Eastern Europe. In: GRODZnisKI W., COWLNG E. B. \& BREYMEYER A. I.

(eds), Ecological risks, perspectives from Poland and the United States, pp. 333-341. National Academy Press, Washington, D.C.

TROJAN P. 1990. Introduction. In: Urban ecological studies in Central and Eastern Europe". Ossolineum, Wroclaw: 5-7. 
Trotan P. 1991. Diptera Tabanidae de Nouvelle Calédonie. Révision des Diachlorini et nouvelles données sur le taons. Mémoirs du Muséum National d'Histoire Naturelle, (A), 149: 251-277.

TROJAN P. 1991. Neubeschreibung von "Tabamus" acallus Szilady, 1926 und seine systematische Stellung innerhalb der Diachlorini (Diptera, Tabanidae). Deutsche Entomologische Zeitschrift, N.F., 38, 4-5: 345-347.

TROJAN P. 1991. Środowisko Polski w perspektywie ekologicznej. In: Człowiek, środowisko, zdrowie". Ossolineum, Wrocław: 71-81.

1992

TROJAN P. 1992. Analiza struktury fauny. Memorabilia Zoologica, 47: 1-121.

\section{3}

TrojaN P. 1993.Über Untersuchungen der Stadtfauna von Warschau. Natur und Landschaft, 68, 10: 531-532.

TROJAN P. 1993. Przedmowa do wydania polskiego. In: MATILE L., TASSY P. \& GOUJET D: "Wstęp do systematyki zoologicznej, koncepcje, zasady, metody". PWN, Warszawa, 107 pp.

TROJAN P. JĘDRYCZKOWSKI W. \& IWAN D. 1993. Zbiory Instytutu Zoologii PAN - zasoby i programy ich opracowania. Rocznik Muzeum Gómośląskiego, Przyroda, 13: 107-111.

TRojaN P. 1993. Próba prognozy zmian różnorodności fauny bezkręgowców lasów Polski dla dwóch scenariuszy zmian klimatycznych. V Sympozjum Ochrony Ekosystemów Leśnych, Streszczenia referatów, Białowieża 18-20. X. 1993 r., 1 p.

\section{4}

Trojan P. 1994. Bogdan Pisarski 1928-1992. Memorabilia Zoologica, 48: 3-7.

TROJAN P. 1994. The shaping of the diversity of invertebrate species in the urban green spaces of Warsaw. Memorabilia Zoologica, 49: 167-173.

TROJAN P. 1994. An attempt at prognosing changes of invertebrate fauna diversity in Polish forests following two scenarios of climate change. V-th Symposium on the Protection of Forest Ecosystems, Warszawa: 267-285.

Trojan P., Bańkowska R., Chudzicka E., Pilipiuk I., Skibínska E., Sterzińska M. \& Wytwer J. 1994. Secondary succession of fauna in the pine forests of Puszcza Białowieska. Fragmenta Faunistica, 37,1: 3-104.

TROJAN P. 1994. Sukcesja wtórna fauny w borach świeżych Puszczy Białowieskiej. Materiały Seminarium "Ochrona Zróżnicowania Biologicznego Ekosystemów Leśnych Puszczy Białowieskiej, Białowieża, 12 pp.

TROJAN P. 1994. Frontoclypeal sutures of some Tabanomorpha (Diptera, Brachycera). Annals of the Upper Silesian Museum, Entomology, 5: 35-51.

TROJAN P. 1994. Supraspecific taxa of Tabaninae (Diptera: Tabanidae). I. The definition of the subfamily and its tribes. Annals of the Upper Silesian Museum, Entomology, 5: 53-67.

TROJAN P. 1994. Supraspecific taxa of Tabaninae (Diptera: Tabanidae). II. The tribe Lepidoselagini and its taxonomic division. Annals of the Upper Silesian Museum, Entomology, 5: 97-172.

\section{5}

Trotan P. \& WYTwER J. 1995. Effect of age differentiation of the pine forest of Puszeza Bialowieska on faunal resources and diversity. Fragmenta Faunistica, 38, 2: 333-338.

TROJAN P. 1995. The lines of secondary succession of fauna in pine forests of the Białowieża Primeval Forest. In: PASCHALIS P., RYKOWSKI K. \& ZAJACZKOWSKI S. (eds), Protection of forest ecosystem biodiversity of Biatowieża Primeval Forest, pp. 153-163. Fundacja "Rozwój SGGW", Warsaw, 272 pp.

TROJAN P. 1995. Occurence of Rhagionidae, Therevidae and Asilidae (Diptera) in the pine forests of the Berezinsky Biosphere Reserve. Fragmenta Faunistica, 38, 9: 187-189.

TROJAN P. 1995. Prognoza zmian różnorodności fauny bezkręgowej lasów Polski dla dwóch scenariuszy zmian klimatycznych. Sylwan, 139, 2: 33-51.

TROJAN P. \& WYTWER J. 1995. Różnorodnośé gatunkowa fauny. In: ANDRZEJEWSKI R. \& WIŚNIEWSKI R. (eds), Problemy różnorodności biologicznej, pp. 37-53. Materiały Konferencji Komitetu "Człowiek i Środowisko", Nauka na rzecz różnorodności biologicznej, Oficyna Wydawnicza Instytutu Ekologii PAN, 124 pp.

\section{6}

TROJAN P. \& WYTWER J. 1996. Różnorodność gatunkowa fauny. In: ANDRZEJEWSKI R. \& WIŚNIEWSKI R. (eds), "Różnorodność biologiczna: Pojęcia, oceny, zagadnienia ochrony i ksztaltowania", Zeszyty Komitetu Naukowego przy Prezydium PAN "Człowiek i Środowisko", Oficyna Wydawnicza Instytutu Ekologii PAN, 15: 39-55.

Trojan P. \& WinIARSKA, G. 1996. Biodiversity studies: First Steps in Poland. 1996. Systematic Biology Network Newsletter, 4: 6.

TROJAN P. 1996. New names for Australian Lepidoselagini (Diptera: Tabanidae). Annals of the Upper Silesian Museum, Entomology, 6-7: 95.

TrojaN P. 1996. The relict forms of Lepidoselagini (Diptera: Tabanidae) in the Holarctic Region. Fragmenta Faunistica, 39, 18: 259-266. 
TROJAN P. 1997. Ocena mozaikowatości środowiska przez analizę dyskryminacyjną rozkładów liczebności gatunków. VI Sympozjum Ekosystemów Leśnych, Jedlnia: 1-10.

TROJAN P., IWAN D. \& WYTWER J. 1997. Morphological relations between the tribes of the subfamily Tabaninae (Diptera: Tabanidae). Polskie Pismo Entomologiczne, 66, 3-4: 277-290.

Trojan P. 1997. Distribution pattern of Diachlorini s. str. (Diptera: Tabanidae). Fragmenta Faunistica, 40, 6: 59-71.

TROJAN P. 1997. Transantarctic relations of Diachlorini s. str. (Diptera: Tabanidae). Fragmenta Faunistica, 40, 13 : $169-189$

Trojan P. 1997. Foreword. In: STerzyńska M,. LeE B. H. \& Trojan P. (eds), Polish-Korean Joint Seminar „Fauna and Flora of the Korean Peninsula: Their Inventory, Systematics and Evolution in Perspective of Biodiversity Conservation", Pultusk, 16-18 September 1996, Poland. Fragmenta Faunistica, 40, 16-31: 205-206.

TROJAN P. 1997. The floristic and faunistic Korean refugium during the last glacial period and its significance in postglacial biota formation. In: STERZYŃSKA M,. LEE B. H. \& TROJAN P (eds), Polish-Korean Joint Seminar „Fauna and Flora of the Korean Peninsula: Their Inventory, Systematics and Evolution in Perspective of Biodiversity Conservation", Pultusk, 16-18 September 1996, Poland. Fragmenta Faunistica, 40: 215-221.

TROJAN P., WYTWER, J. 1997. Numerical methods of biodiversity studies and the problems of the protection of nature. In: STERZYNSKA M, LEE B. H. \& TROJAN P (eds), Polish-Korean Joint Seminar „Fauna and Flora of the Korean Peninsula: Their Inventory, Systematics and Evolution in Perspective of Biodiversity Conservation', Pultusk, 16 18 September 1996, Poland. Fragmenta Faunistica, 40: 223-230.

\section{8}

TROJAN P. 1998. Dwie hipotezy o rozprzestrzenianiu się gondwańskich Tabanidae a datowanie dryftu kontynentów. Dipteron, 14: 15. Polskie Towarzystwo Entomologiczne, Sekcja Dipterologiczna, Gdańsk-Lódź.

TROJAN P. 1998. Nowe perspektywy w badaniach entomofaunistycznych. New prospects in entomofaunistic studies. Wiadomości Entomologiczne, 17 Suppl.: 137-155.

TROJAN P. 1998. Supraspecific taxa of Tabaninae (Diptera: Tabanidae). III. The tribe Diachlorini and its taxonomic division. Annals of the Upper Silesian Museum, Entomology, 8-9: 5-91.

TROJAN P. 1998. Bioróżnorodność jako kryterium jakości środowiska naturalnego czlowieka. Problemy środowiska $\mathrm{i}$ jego ochrony, Katowice, 6: 65-80.

TROJAN P. 1998. Faunistic studies in Poland - historiography and stages of development. Fragmenta Faunistica, 41,10 $139-150$.

\section{0}

TROJAN P. 2000. The meaning and measuring of species diversity. Fragmenta Faunistica, 43, 1: 1-12.

TROJAN P. 2000. Wyznaczanie gatunków owadów zagrożonych wyginięciem. Wiadomości Entomologiczne, 18 , Suppl. 2: 221-232.

Trojan P. \& DRABer-MoNko A. 2000. Doktor Muchołapski - fikcja literacka, czy postać rzeczywista. Notatki Entomologiczne, 1, 3: 82-87. Wydawnictwo Mantis, Olsztyn.

\section{1}

TRoJAN P. 2001. Kłopoty nauk ekologicznych. Dziś, 12, 9: 52-57.

TROJAN P. 2001. Ekologia w Polsce: czy diagnoza i czy terapia? Nauka, 3: 105-122.

TrojaN P. 2001. Rhagionidae, Coenomyiidae, Xylophagidae, Xylomyiidae, Stratiomyidae, Tabanidae, Asilidae, Scenopinidae, Bombyliidae. In: GuTOWski J. M. \& JARoszewicz B. (eds), Katalog fauny Puszczy Białowieskiej, Instytut Badawczy Leśnictwa, Warszawa: 278-282.

TrojaN P. 2001. Banaszak J., Wiśniewski H. Podstawy ekologii. Wydawnictwo Uezelniane WSP w Bydgoszezy, 1999, 630 pp. [ISBN 83-7096-296-3]. Wiadomości Ekologiczne, 47, 3: 238-242.

Trotan P. 2001. Supraspecific taxa of Tabaninae (Diptera: Tabanidae). IV. The tribe Tabanini and its taxonomic division. Annals of the Upper Silesian Museum, Entomology, 10-11: 31-62.

TROJAN P. \& WINIARSKA G. 2001. Miasto jako archipelag wysp śródlądowych. In: INDYKIEWICZ P., BARCZAK T. \& KACZOROWSKI G. (eds), Bioróżnorodność i ekologia populacji zwierzęcych w środowiskach zurbanizowanych, Nice, Bydgoszcz: 11-16.

TROJAN P. 2001. Hasła: agrocenoza, agroekologia, akumulacja biologiczna substancji, algofagi, Alleego zasada, amensalizm. Wielka Encyklopedia PWN, 1: 213, 293, 384, 403, 471

TROJAN P. 2001. Hasła: arenal, astatyczne środowisko, atmofile, autekologia, autotrofy. Wielka Encyklopedia PWN, 2 : $261,376,435,526,544$.

TROJAN P. 2001. Hasła: biocenologia, biogeocenologia, bionomiczna strategia, biota, biotop, biotyczny potencjal, briofile. Wielka Encyklopedia PWN, 4: 77, 85-86, 98-99, 103, 105, 106, 445.

TROJAN P. 2001. Hasła: cenoza, chronobiologia. Wielka Encyklopedia PWN, 5: 256, 543. 
TROJAN P. 2002. Special issue, a tribute to the Dipterological Laboratory (1956-1975) of the Institute of Zoology PAS and the associated dipterologists. Annales Zoologici, 52 (2): 189-193.

TROJAN P. 2002. First discovery of Bouvieromyiini (Diptera: Tabanidae, Chrysopsinae) in Baltic amber. Annales Zoologici, 52, 2: 257-270.

TROjAN P. \& SMOLEŃSKi M. 2002. 16. Ocena różnorodności gatunkowej w złożonych układach ekologicznych. In: BANASZAK J. (ed.), Wyspy środowiskowe. Bioróżnorodność i próby typologii, Wydawnictwo Akademii Bydgoskiej im. K. Wielkiego, Bydgoszcz: 285-299.

TROJAN P., SMOLENSSK M. \& WYTWER J. 2002. Teoria homeostazy i sukcesji ekologicznej a różnorodność gatunkowa fauny borów sosnowych. In: SŁAWSKA M. \& SMOLEŃSKI M., VII Sympozjum Ochrony Ekosystemów Leśnych, Wydawnictwo SGGW: 32-54.

TROJAN P. 2002. Hasła: deficyt wodny roślin, degradacja ekosystemów, democen, demografia ekologiczna, dominacja, dominanty, drapieżnictwo. Wielka Encyklopedia PWN, 7: 18, 24, 68, 70, 280-281, 337-338.

TROJAN P. 2002. Hasła: edaficzne czynniki, edafon, edafotop, edyfikatory, ekofizjologia, ekogeograficzne reguły, ekoklimat, ekoklina, ekologia, ekologia populacyjna, ekologiczna bariera, ekologiczna eksplozja, ekologiczna energetyka, ekologiczna genetyka, ekologiczna izolacja, ekologiczna konkurencja, ekologiczna kooperacja, ekologiczna piramida, ekologiczna wierność, ekologiczna wydajność, ekologiczne czynniki, ekologiczne modele, ekologiczny korytarz, ekologizm, ekosystem, ekoton, ekstremofile, ekstynkcja, energetyczne budżety. Wielka Encyklopedia PWN, 8: 34-35, 45, 111-114, 115-116, 117, 124-125, 138, 139, 257.

TrojaN P. 2002. Hasło: fizjocenoza, Gauzego zasada. Wielka Encyklopedia PWN, 9: 163, 561.

TRoJAN P. 2002. Hasla: geokompleks, gradocen, fizjocenoza. Wielka Encyklopedia PWN, 10: 76, 370.

TROJAN P. 2002. Hasła: habitat, hematofagi, homeostaza ekosystemu. Wielka Encyklopedia PWN, 11: 58, $223,437$.

TROJAN P. 2002. Hasła: industrioklimaks, influenty, integracjonizm, interakcje międzygatunkowe, izocenozy. Wielka Encyklopedia PWN, 12: 112, 126, 176, 186, 313.

\section{3}

TROJAN P. 2003. Hasło: klimaks. Wielka Encyklopedia PWN, 14: 27.

TROJAN P. 2003. Hasła: liczebnośé, Liebiga prawo minimum. Wielka Encyklopedia PWN, 15: 543, 549-550.

TROJAN P. 2003. Hasła: Lincolna wskaźnik, Lotki - Volterry równania, łańcuchy pokarmowe. Wielka Encyklopedia PWN, 16: 20, 147, 261.

TROJAN P. 2003. Hasła: merotop, Międzynarodowy Program Biologiczny. Wielka Encyklopedia PWN, 17: $268,391$.

TROJAN P. 2003. Hasła: monotop, mozaikowatość środowiska. Wielka Encyklopedia PWN, 18: 65, 152.

\section{4}

Trojan P. 2004. Współczesne powiązania ślepaków (Diptera: Tabanidae) z bursztynu baltyckiego. Prace Muzeum Ziemi, 47: 87-88.

Trojan P. 2004. Hasło: nisza ekologiczna. Wielka Encyklopedia PWN, 19: 78.

TROJAN P. 2004. Hasła: osiadle osobniki; ostoja, refugium. Wielka Encyklopedia PWN, 20: 55, 77.

TROJAN P. 2004. Hasła: relikt, rozkłady przestrzenne organizmów. Wielka Encyklopedia PWN, 23: 245-246, 554.

TROJAN P. 2004. Hasła: równowaga biocenotyczna, różnorodność biologiczna, samoregulacja, seralne stadia. Wielka Encyklopedia PWN, 24: 18, 30-32, 298, 534.

Banaszak J., Buszko J., Czachorowski S., Czechowska W., Hebda G., Liana A., Pawlowski J., SzePt YCKi A., TROJAN P. \& WĘGEREK P. 2004. Przegląd badań inwentaryzacyjnych nad owadami w parkach narodowych Polski. Wiadomości Entomologiczne, 23 Supl. 2: 5-56.

\section{5}

TROJAN P. 2005. Hasła: Shannona-Wienera miara, siedlisko, Simpsona miara. Wielka Encyklopedia PWN, 25: 12, 47, 97.

TROJAN P. 2005. Hasła: stepowienie krajobrazu, subdominanty, sukcesja ekologiczna, synantropijne zwierzęta, synantropizacja, synekologia. Wielka Encyklopedia PWN, 26: 58, 193, 223, 317, 320.

TROJAN P. 2005. Hasła: środowisko biologiczne, środowisko przyrodnicze, tabele przeżywania. Wielka Encyklopedia PWN, 27: 48, 100.

TROJAN P. 2005. Hasło: troficzny poziom. Wielka Encyklopedia PWN, 28: 39.

TROJAN P. 2005. Hasła: zasięgi organizmów, zastępstwo ekologiczne, zdjęcie fitosocjologiczne, zespół ekologiczny, ziemne organizmy, zoocenologia, zoocenoza. Wielka Encyklopedia PWN, 30: 274-275, 279, 314, 335, $371,432$.

\section{7}

TROJAN P. 2007. Rediscovery of an „extinct” fly Adapsilia coarctata Waga, 1842 (Diptera: (Pyrgotidae) in Poland, Fragmenta Faunistica, 50 (1): 43-46.

Trojar P. 2007. Muchówki, Dwuskrzydłe (Diptera), pp. 9-12. In: Bogdarowicz W., Chudzicka E., PILIPIUK I. \& SKIBIŃSKA E. (eds), Fauna Polski - charakterystyka i wykaz gatunków [Fauna of Poland - characteristics and checklist of species]. Muzeum i Instytut Zoologii PAN, Warszawa, 2, 505 pp. 
Trojar P. 2007. Krótkorogie (Brachycera), pp. 79-82. In: Bogdanowicz W., Chudzicka E., PILIPIUK I. \& SKIBINSKA E. (eds), Fauna Polski - charakterystyka i wykaz gatunków [Fauna of Poland - characteristics and checklist of species]. Muzeum i Instytut Zoologii PAN, Warszawa, 2, 505 pp.

Trojan P. 2007. Athericidae, pp. 82-83, 175. In: Bogdanowicz W., ChUdzicka E., PilipIuK I. \& SkiBIńsKa E. (eds), Fauna Polski - charakterystyka i wykaz gatunków [Fauna of Poland - characteristics and checklist of species]. Muzeum i Instytut Zoologii PAN, Warszawa, 2, 505 pp.

Trojan P. 2007. Ślepaki (Tabanidae), pp. 83-84, 175-176. In: Bogdanowicz W., Chudzicka E., PLIPIUK I. \& SKIBIjSKA E. (eds), Fauna Polski - charakterystyka i wykaz gatunków [Fauna of Poland - characteristics and checklist of species]. Muzeum i Instytut Zoologii PAN, Warszawa, 2, 505 pp.

Trojan P. 2007. Lwinkowate (Stratiomyidae), pp. 84-85, 176-177. In: Bogdanowicz W., Chudzicka E., PiLIPIUK I. \& SKIBIŃSKA E. (eds), Fauna Polski - charakterystyka i wykaz gatunków [Fauna of Poland - characteristics and checklist of species]. Muzeum i Instytut Zoologii PAN, Warszawa, 2, 505 pp.

Trojan P. 2007. Xylomyidae, pp. 85, 177. In: Bogdanowicz W., ChUdzICKA E., PLIPIUK I. \& SkIBINSKA E. (eds), Fauna Polski - charakterystyka i wykaz gatunków [Fauna of Poland - characteristics and checklist of species] Muzeum i Instytut Zoologii PAN, Warszawa, 2, $505 \mathrm{pp.}$

TROJAN P. 2007. Kobyliczkowate (Rhagionidae), pp. 85-86, 177. In: BOGDANOWICZ W., CHUDZICKA E., PILIPIUK I. \& SKIBnjsKA E. (eds), Fauna Polski - charakterystyka i wykaz gatunków [Fauna of Poland - characteristics and checklist of species]. Muzeum i Instytut Zoologii PAN, Warszawa, 2, 505 pp.

Trojan P. 2007. Coenomyiidae, pp. 86-87, 177. In: BogdanowiCz W., ChUdzICKa E., PILIPIUK I. \& SkIBIŃSKA E. (eds), Fauna Polski - charakterystyka i wykaz gatunków [Fauna of Poland - characteristics and checklist of species]. Muzeum i Instytut Zoologii PAN, Warszawa, 2, 505 pp.

TrojaN P. 2007. Xylophagidae, pp. 87, 177. In: BogdANOWICZ W., ChUdzICKA E., PLIPIUK I. \& SkIBníska E. (eds), Fauna Polski - charakterystyka i wykaz gatunków [Fauna of Poland - characteristics and checklist of species]. Muzeum i Instytut Zoologii PAN, Warszawa, 2, 505 pp.

Trojan P. 2007. Lowiki Asilidae, pp.87-89, 178-179. In: Bogdanowicz W., Chudzicka E., PILIPIUK I. \& SKIBIjSKA E. (eds), Fauna Polski - charakterystyka i wykaz gatunków [Fauna of Poland - characteristics and checklist of species]. Muzeum i Instytut Zoologii PAN, Warszawa, 2, 505 pp.

Trojan P. 2007. Zmrużkowate (Scenopinidae), pp. 89, 179. In: Bogdanowicz W., Chudzicka E., PLIPIUK I. \& SKIBIjSKA E. (eds), Fauna Polski - charakterystyka i wykaz gatunków [Fauna of Poland - characteristics and checklist of species]. Muzeum i Instytut Zoologii PAN, Warszawa, 2, 505 pp.

TROJAN P. 2007. Dziewierkowate (Therevidae), pp. 89-90, 179-180. In: BoGDANOWICZ W., CHUDZICKA E., PILIPIUK I. \& SKIBnjSKA E. (eds), Fauna Polski - charakterystyka i wykaz gatunków [Fauna of Poland - characteristics and checklist of species]. Muzeum i Instytut Zoologii PAN, Warszawa, 2, 505 pp.

Trojan P. 2007. Acroceridae, pp. 90-91, 180. In: BOGDANOWICZ W., CHUDZICKA E., PILIPIUK I. \& SKIBIŃSKA E. (eds), Fauna Polski - charakterystyka i wykaz gatunków [Fauna of Poland - characteristics and checklist of species]. Muzeum i Instytut Zoologii PAN, Warszawa, 2, 505 pp.

Trojan P. 2007. Bujanki (Bombyliidae), pp. 91-92, 180. In: Bogdanowicz W., Chudzicka E., PILIPIUK I. \& SKIBIŃSKA E. (eds), Fauna Polski - charakterystyka i wykaz gatunków [Fauna of Poland - characteristics and checklist of species]. Muzeum i Instytut Zoologii PAN, Warszawa, 2, 505 pp.

\section{8}

TROJAN P. 2008. Przedmowa. In: BANASZAK J. „Czas nie przeszedt obok. Wspomnienia przyrodnika”. Oficyna Wydawnicza Branta: 7-9.

TROJAN P. 2008. Stabilność ekosystemów a zróżnicowanie biologiczne - najnowsze poglądy i koncepcje. In: MAZUR S. \& TRACZ H. (eds), VIII Sympozjum Ochrony Ekosystemów Leśnych - Zagrożenia Ekosystemów Leśnych przez Człowieka, Rozpoznanie - Monitoring - Przeciwdziałanie: 19-29.

\section{9}

TROJAN P. 2009. Two species of supranivale insects new for Kampinos National Park. Fragmenta Faunistica 52: 207 208. 


\section{PROFESSOR PRZEMYSLAW TROJAN - ON HIS 80TH BIRTHDAY}

Przemysław Trojan, Professor Emeritus at the Polish Academy of Sciences Museum and Institute of Zoology, celebrated his $80^{\text {th }}$ birthday in 2009 . Of these eighty years, fifty-five have been devoted to research in biology. A session of the Institute's Scientific Council held on 8 October 2009 was followed by a ceremony to honour this jubilee. Professor Trojan's profile as a scientist was presented to the members of the Council and the invited guests by Prof. Stanisław L. Kazubski, Vide-President of the Scientific Council. The early years of his creative work at the Institute of Zoology were brought back by Dr Waldemar Mikołajczyk, who emphasised Prof. Trojan's contribution to the development of dipteran studies in Poland. Dr Jolanta Wytwer provided a short summary of his academic achievements and his contribution to faunology and general ecology. Prof. Andrzej Szujecki described Prof. Trojan's contribution to the development of research in forest ecology. Following these presentations, Professor Przemysław Trojan received a commemorative medal and a bouquet of roses from Director of Museum and Institute of Zoology Prof. Wiesław Bogdanowicz. All those present were subsequently treated to a glass of champagne and a slice of a three-tiered layer cake.

Editorial Board of Fragmenta Faunistica

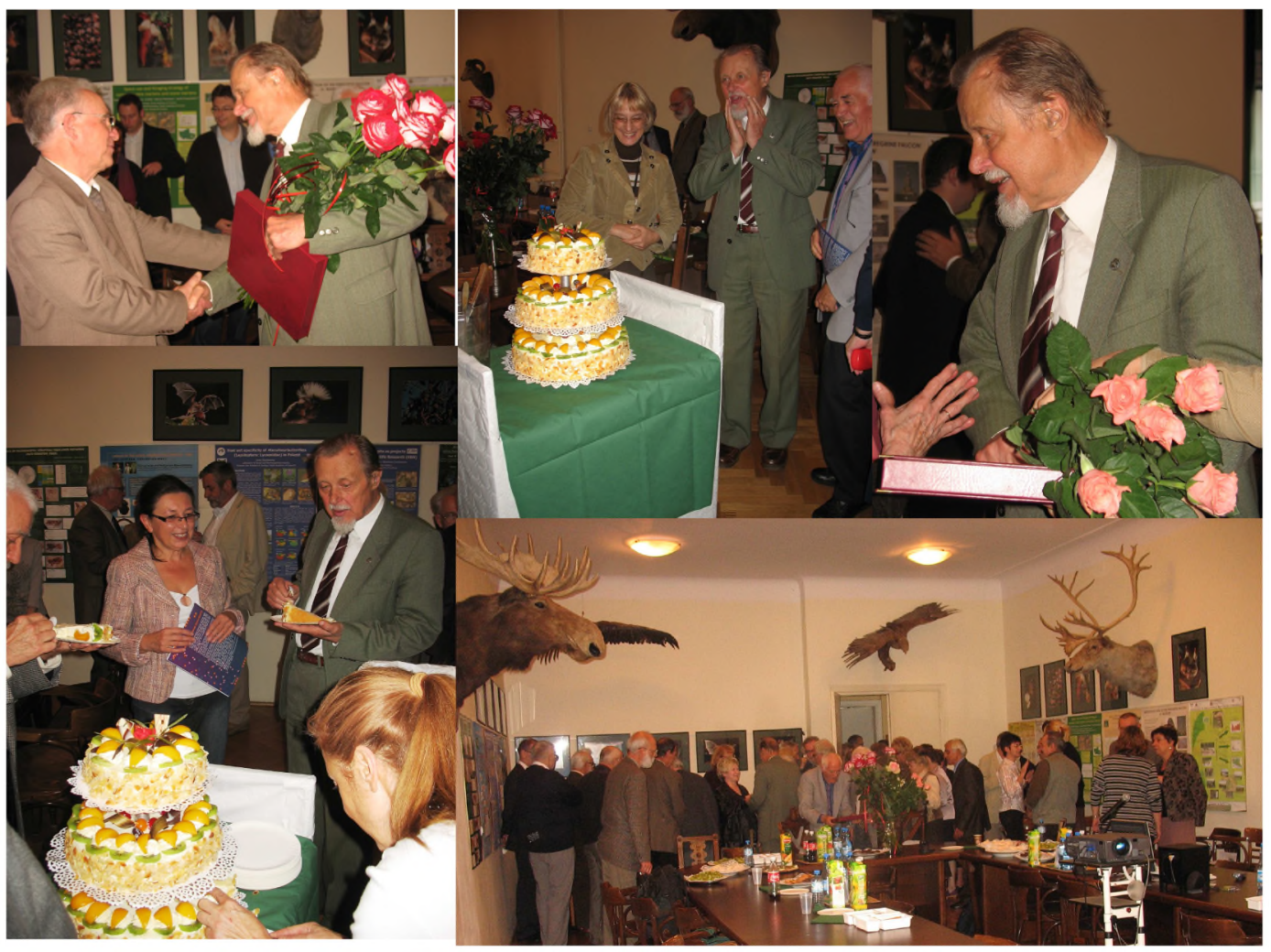


Dr Waldemar Mikolajezyk. I met Professor Trojan for the first time 55 years ago. As a second-year student at Warsaw University's Faculty of Biology, I had begun voluntary work at the then Polish Academy of Sciences Zoological Institute. I was assigned to the Dipterological Laboratory, which Prof. Trojan was organising at that time. The laboratory was the second largest unit of the Insect Division, following the coleopterological laboratory. It was staffed by a team of young researchers and technicians, all nearly of the same age. The head, Przemyslaw Trojan, MSc, knew well how to engage everyone in joint projects, from the drafting of collective and individual research plans, to reviewing and completing the literature base, arranging existing collections and creating new ones, and brainstorming ideas for the purchase of technical equipment.

His research initiatives, both at this early stage and later, very soon bore fruit in the form of numerous publications in dipteran research, leading to improved knowledge of dipterans, mostly, though not exclusively, from Poland. The young manager never refused to help, readily shared his knowledge and experience and would respect the views of others even if he sometimes did not share these views. He had a vision of the Laboratory's future development and tasks that it should fulfil, with emphasis on continuation of the work of prominent Warsaw dipterologists of the 19th century, predominantly Jan Sznabl and Henryk Dziedzicki. Thanks to these efforts, the Institute of Zoology became an important centre of dipteran studies in Poland. Prof. Trojan himself has provided a more extensive account (Trojan P. 2002: Historical remarks. In: Special issue, a tribute to the Dipterological Laboratory (1956-1975) of the Institute of Zoology PAS and the associated dipterologists. Annales Zoologici, 52 (2): 189-193).

Professor Trojan always found it easy to take decisions, draw conclusions, provide synthetic views, and write. The scope of his research interests went far beyond the disciplines traditionally associated with the Dipterological Laboratory, i.e. faunal studies, taxonomy or systematics. His diverse interests naturally influenced the course of his academic career.

Dr Jolanta Wytwer. Taxonomy and dipterology represent just one part of the wide range of Professor Trojan's academic interests. Of the almost 300 publications, more than 200 are concerned with other areas of biology. Professor Trojan's wide range of interests always led to the publication of important academic deliverables, initially in the form of course books or articles or even popular science books (Muchy $i$ czlowiek [Flies and Man]), and in time they became a separate thread in his research activity. Two realms - ecology and faunal studies are particularly important in this respect.

The generations of biology students from the 1970's and 1980's will well remember Ekologia Ogólna [General Ecology], first published in 1975, the first textbook in ecology ever written by a Polish writer. The textbook was not only the result of Professor Trojan's developing interest in the theory of ecology, but also drew on his growing experience related, among others, to research undertaken at the PAS Agroecology Unit, which he organised in Turew near Poznań, as well as his teaching commitments at the Faculty of Biology of the Silesian University in Katowice, where he was a co-founder and organiser of the first degree programme in ecology in Poland.

Separate volumes were published discussing in detail such issues in ecology as Homeostaza ekosystemów [Ecosystem Homoeostasis] (1980) and Bioklimatologia ekologiczna [Ecological Bioclimatology] (1985). The former, available also in English, is a succinct and lucid presentation of a novel approach to ecosystem homoeostasis and still remains the most comprehensive and complete analysis of the concept of ecosystem stability written by a Polish author. 
All three textbooks are still read by students of various courses in ecology and climate science at Polish universities, continuing to serve their educational purposes for those who buy them both from traditional old book shops and on-line.

This novel approach to topics in ecology, presented, among others, in Homeostaza ekosystemów, revolved chiefly around the concept of biodiversity at species level, which Prof. Trojan analysed mainly in agrocoenoses and in ecological studies of urban faunas. These issues, approached from various angles (trophic dynamics, faunal studies, zoogeography and with regard to the phenomenon of synanthropisation), accounted for the majority of research undertaken at the Institute of Zoology in the 1970's, winning prestige and awards for our institution. Extensive studies of invertebrate assemblages in urban environments were very innovative and brought considerable publicity and recognition to the Institute (see issues of Memorabilia Zoologica from the period 1981-1989). We know now that this research formed mainstream biodiversity studies in Poland in that period.

As this research developed, the idea of identifying typical faunal structures was born. The outcomes of this approach included the development of methods of analysis of faunal structures (Analiza struktury fauny, [Analysis of Faunal Structure] 1992). An extended methodology of faunal research was used in studies of secondary succession of the fauna of pine forests in Białowieża Forest, the results of which were published in Fragmenta Faunistica in 1994, bringing another award for the Institute's research team, which I had the pleasure of being a member of at that time.

Professor Trojan's work on Diptera cannot be legitimately separated from his efforts to unravel ecological processes and identify faunal structures, since, closely intertwined, all these research orientations stimulated each other and added to the achievements of Polish science in taxonomy, ecology and fauna studies.

Prof. Andrzej Szujecki. I first met Przemyslaw Trojan, then a research assistant working with Prof. Marian Gieysztor, in early 1950, at the Department of Zoology of Warsaw Agricultural University, where I, a student of forestry, was attending practical classes. Soon afterwards, having obtained consent from the Vice-Director of the Museum of Zoology in Warsaw Prof. Janusz Nast to do voluntary work there, I was working on the $5^{\text {th }}$ floor in the beetle section under the supervision of Custodian Józef Makólski. There I met the future Professor Przemysław Trojan, who was studying the Institute's dipteran collections one floor below. Since then I have always been in awe of his extensive knowledge of biology. As an ecologist, Professor Trojan has exerted a powerful influence on the development of this discipline of science, including both university education and the orientation of research activity.

With regard to ecology, his particular research interests have included:

- identification of faunal structures,

- identification of changes in these structures in the course of ecological succession and in response to stress, and

- the concept of homoeostasis of ecological systems and conditions for achieving homoeostasis.

His studies of faunal structures initially concentrated on assemblages of tabanid flies, but were later extended to include other groups of heteropteran and homopteran insects.

The papers "Nisze ekologiczne różnych gatunków Tabanidae w Puszczy Kampinoskiej" [Ecological Niches of Various Tabanid Species in Kampinos Forest] (1958), "Struktura zgrupowań jako wskaźnik homeostazy ekosystemu" [Assemblage Structure as Indicator of Ecoystem Homoeostasis] (1980), "Tabaninomorpha Warszawy i Mazowsza" [Tabaninomorpha of Warsaw and Mazovia] (1981), "Zgrupowania Heteroptera w krajobrazie rolniczym 
Wielkopolski" [Heteropteran Aseemblages in Agricultural Landscape of Great Poland] (1989) not only contain methodological and statistical considerations that are indispensable in descriptions of faunal structures, but also link elements of structural description with ecosystem properties, namely, homoeostasis. The author also analysed the scope of faunal variation against the background of man-induced environmental transformations. He went on to present a summary of his research, and confront his data with a rich body of foreign studies, in his excellent monograph "Analiza struktury fauny" [Analysis of Faunal Structure] (1992), a work which became a methodological guide for vast numbers of faunal researchers and ecologists studying faunal assemblages.

The year 1980 saw the publication of an earlier work titled "Homeostaza ekosystemów" [Homoeostasis of Ecosystems], which represented a breakthrough in the identification of feedback mechanisms operating in multispecies systems. In this work, the author formulated, and supplied suitable illustrative examples for, four principles of ecological homoeostasis (presented previously in his textbook "Ekologia ogólna" [General Ecology] in 1975):

- the principle of preservation of structure,

- the principle of preservation of the flow of matter,

- the principle of preservation of productivity, and

- the principle of stabilisation of ecosystem processes.

An important experimental foundation underlying the formulation of these principles was research on Acyrtosiphon aphids, feeding on alfalfa, carried out by the research team of the PAS Institute of Zoology in the 1970's. These investigations revealed that the structure of the faunal assemblages did not resemble a pyramid but represented a complex system that included, apart from the sequence of links in the food chain, elements operating in parallel with the food chain that performed regulatory functions at systemic level.

Other studies related to the aforementioned dissertation focused mainly on the relation between structural models and homoeostasis in communities, with particular attention paid to the effects of environmental transformations. Published reports on the results of these studies included "Proces synantropizacji w konkurencyjnych zespołach zwierząt" [Synanthropisation in Competivie Animal Communities] (co-authored with Górska and Wegner, 1982), "Zmiany różnorodności gatunków bezkręgowców terenów zielonych w Warszawie" [Diversity changes among Invertebrate Species Inhabiting Green Areas in Warsaw] (1994), "Ocena mozaikowatości środowisk przez analizę dyskryminacyjną rozkładów liczebności gatunków" [Assessment of Habitat Patchiness by Discriminant Analysis of Species Abundance Distributions] (1997), and "Stabilność ekosystemów a zróżnicowanie biologiczne - najnowsze poglądy" [Ecosystem Stability and Biological Diversity - Latest Views] (2008). Prof. Trojan stated in them that any equilibrium observed in nature is momentary and depends on the relations between all species forming the community. Stress leads to a reduction in the number of species and changes in the assemblage's structural pattern. Urbanisation pressure, for instance, results primarily in the elimination of species of narrow ecological tolerance, so that only eurytopic and poytopic species can be found in city centres.

In his presentation during a 1998 Symposium in Bialowieża (1998), Prof. Trojan named five factors that contribute to the degradation of forest fauna:

- environmental unification, i.e. the presence of one type of habitat in large areas,

- homogeneity of habitats as a result of destruction of their vertical structure,

- simplification of biocoenoses through the introduction of monocultures,

- environmental intoxication by noxious immissions,

- aridification of the environment following elimination of the litter layer. Faunal responses to these stressors comprise the following: 
- changes in the status of dominant species,

- uneven reductions in individual trophic groups, and

- an increasing proportion of eurybionts.

This knowledge of assemblage responses to environmental stress allowed Prof. Trojan to develop two scenarios of expected changes in the structure and diversity of the fauna in the context of predicted climate change. One of these scenarios assumes a dry warm climate setting, whereas the other one is concerned with a moist warm climate. In both cases, the most prominent faunal changes would be seen in pine forests, with broad-leaved forests registering less significant change ( $5^{\text {th }}$ Forest Ecosy stem Protection Symposium).

The totality of outcomes of Prof. Trojan's extensive studies of community structure and community homoeostasis is not to be overestimated. Let me, however, voice my rather subjective view that he obtained equally valuable and interesting, and perhaps also the most revolutionary, results when he guided and systematised the results of the Institute's team work on assemblage succession, and particularly assemblages of epiphytic insects in the pine stands of Białowieża Forest $(1994,1995)$. These investigations demonstrated that the structure of ecosystems in nature is more complex that idealised theoretical models had attempted to show. Studies of 30 faunal groups revealed the presence of as many as five types of succession (creative, stabilising, 'broken stick', regressive, restorative). A significant conclusion arising from studies of the various patterns of secondary succession is that secondary succession is usually associated with decreases in both species numbers and biodiversity indices, while the number of individuals actually increases and the dominance differences become sharper. These achievements are not only important for theory of ecology since they represent verification of the models of succession proposed by Odum and Margalef, but they may also be applicable to the practice of ecosystem regulation, or ecological engineering. They bring home the truth that ecosystems of the temperate (and boreal) zone are inherently characterised by variability of states of nature, and that the homoeostatic value of an ecosystem does not depend on permanent stability but on the ability to restore a previous state following a disturbance, i.e. ecosystem resilience. This ability depends on the most abundant species in the community as well as on so-called "key" species, whose presence in the structure of the ecosystem is consolidated in the course of succession, as species that are not necessary for the organisation of final (climax) communities are sifted out.

It is not possible, given the limited time, to present Professor Trojan's contribution to the advancement of knowledge, the development of various academic centres, but I have the pleasure, and an obligation, of emphasising his collaboration with the Department of Forest Protection and Ecology of Warsaw Agricultural University, which included co-participation in research programmes and the immense influence that Prof. Trojan exerted by presenting the results of his work and state-of-the-art ecological theories during consecutive Symposia for the Protection of Forest Ecosystems organised by the Department. In doing this, he influenced the development of a pro-ecological model of forest economy. For this I thank him wholeheartedly. I congratulate him on such significant achievements and wish continued satisfaction in his work for science. 